\title{
Electromagnetic conic sections
}

Tevian Dray a)

Department of Mathematics, Oregon State University, Corvallis, Oregon 97331

Corinne A. Manogue ${ }^{\text {b) }}$

Department of Physics, Oregon State University, Corvallis, Oregon 97331

(Received 29 May 2001; accepted 14 June 2002)

Certain orthogonal coordinate systems naturally correspond to basis vectors which are both curl-free and divergence-free, and hence solve Maxwell's equations. After first comparing several different traditional approaches to computing div, grad, and curl in curvilinear coordinates, we present a new approach, based on these "electromagnetic" basis vectors, which combines geometry and physics. Not only is our approach tied to a physical interpretation in terms of the electromagnetic field, it is also a useful way to remember the formulas themselves. We give several important examples of coordinate systems in which this approach is valid, in each case discussing the electromagnetic interpretation of the basis. We also give a general condition for when an electromagnetic interpretation is possible. (c) 2002 American Association of Physics Teachers.

[DOI: $10.1119 / 1.1501115]$

\section{INTRODUCTION}

What are the divergence and curl of a vector field? Students in mathematics courses often learn algebraic formulas for these derivatives, without learning the geometry behind them. Those students who go on to take physics or engineering courses which use these concepts often have trouble "bridging the gap" between the way vector calculus is taught by mathematicians and the way it is used in applications. ${ }^{1,2}$ One indication of the extent of this problem is the fact that few mathematicians have seen the notation $\{\hat{\mathbf{r}}, \hat{\boldsymbol{\theta}}, \hat{\boldsymbol{\phi}}\}$ for the unit vectors for spherical coordinates, yet most physicists assume that their students learn this in vector calculus. ${ }^{3}$ This problem is exacerbated by the different conventions for spherical coordinates used by physicists and mathematicians.

We emphasize that the use of nonrectangular coordinate bases is just one small step; the gap must be bridged at a more fundamental level. ${ }^{4}$ And a few simple examples, such as spherical coordinates, are sufficient for, say undergraduate physics. The goal of this paper is rather to consider several more sophisticated examples, showing explicitly how they relate to electromagnetism.

Why are the formulas for divergence and curl so much harder in curvilinear coordinates than in rectangular coordinates? Because the basis vectors are not constant. However, if the basis vectors were both curl-free and divergence-free, they would pull through the computation of the curl and divergence as though they were constant, dramatically simplifying things. This is the basic idea we will develop here. Furthermore, a (time-independent) vector field which is both curl-free and divergence-free solves Maxwell's equations; such vector fields correspond to an electromagnetic field in vacuum.

We begin by reviewing several traditional ways of computing div, grad, and curl in curvilinear coordinates, and in particular contrasting the approaches used by mathematicians and physicists. We then consider several important examples which naturally correspond to an "electromagnetic basis," in each case discussing the electromagnetic interpretation. Most of the examples we give are special cases of ellipsoidal coordinates; we call them electromagnetic conic sections. We then give a necessary and sufficient condition for a given vector field to be electromagnetic, that is, to admit a rescaling which is both curl-free and divergencefree. Finally, we show how to use these basis vectors to simplify the computation of div, grad, and curl.

\section{CALCULATING DIV, GRAD, AND CURL}

\section{A. Mathematics}

In introductory mathematics courses, one typically works in Cartesian coordinates, using the basis $\{\hat{\mathbf{i}}, \hat{\mathbf{j}}, \hat{\mathbf{k}}\}$. Given any vector field

$$
\overrightarrow{\mathbf{F}}=P \hat{\mathbf{i}}+Q \hat{\mathbf{j}}+R \hat{\mathbf{k}}
$$

the divergence and curl of $\overrightarrow{\mathbf{F}}$ are defined by the formulas

$$
\vec{\nabla} \cdot \overrightarrow{\mathbf{F}}=\frac{\partial P}{\partial x}+\frac{\partial Q}{\partial y}+\frac{\partial R}{\partial z}
$$

and

$$
\vec{\nabla} \times \overrightarrow{\mathbf{F}}=\left|\begin{array}{ccc}
\hat{\mathbf{i}} & \hat{\mathbf{j}} & \hat{\mathbf{k}} \\
\frac{\partial}{\partial x} & \frac{\partial}{\partial y} & \frac{\partial}{\partial z} \\
P & Q & R
\end{array}\right|,
$$

respectively. It is an instructive exercise to try to use these formulas to verify that

$$
\vec{\nabla} \cdot \frac{(x \hat{\mathbf{i}}+y \hat{\mathbf{j}}+z \hat{\mathbf{k}})}{\left(x^{2}+y^{2}+z^{2}\right)^{3 / 2}}=0
$$

(the charge density for a point charge, away from the source) and that

$$
\overrightarrow{\boldsymbol{\nabla}} \times \frac{(x \hat{\mathbf{j}}-y \hat{\mathbf{i}})}{\left(x^{2}+y^{2}\right)}=\overrightarrow{\mathbf{0}}
$$

(the current density of a line charge, away from the source). These are perhaps the two most important elementary physical examples of divergence and curl. 


\section{B. Product rules}

An important simplification occurs by using basis vectors adapted to the symmetry of the problem. Introducing the spherical basis vectors

$$
\begin{aligned}
& \hat{\mathbf{r}}=\sin \theta \cos \phi \hat{\mathbf{i}}+\sin \theta \sin \phi \hat{\mathbf{j}}+\cos \theta \hat{\mathbf{k}}, \\
& \hat{\boldsymbol{\theta}}=\cos \theta \cos \phi \hat{\mathbf{i}}+\cos \theta \sin \phi \hat{\mathbf{j}}-\sin \theta \hat{\mathbf{k}}, \\
& \hat{\boldsymbol{\phi}}=-\sin \phi \hat{\mathbf{i}}+\cos \phi \hat{\mathbf{j}},
\end{aligned}
$$

it is straightforward but messy to use (2) and (3) to calculate, for instance, that

$$
\begin{aligned}
& \overrightarrow{\boldsymbol{\nabla}} \cdot \hat{\mathbf{r}}=\frac{2}{r}, \\
& \overrightarrow{\boldsymbol{\nabla}} \times \hat{\boldsymbol{\phi}}=\frac{\cot \theta}{r} \hat{\mathbf{r}}-\frac{1}{r} \hat{\boldsymbol{\theta}}
\end{aligned}
$$

either using the chain rule, or by rewriting $(r, \theta, \phi)$ in terms of $(x, y, z)$. If one now recalls that vector differentiation satisfies the product rules

$$
\begin{aligned}
& \vec{\nabla} \cdot(f \overrightarrow{\mathbf{F}})=\vec{\nabla} f \cdot \overrightarrow{\mathbf{F}}+f(\vec{\nabla} \cdot \overrightarrow{\mathbf{F}}), \\
& \vec{\nabla} \times(f \overrightarrow{\mathbf{F}})=\vec{\nabla} f \times \overrightarrow{\mathbf{F}}+f(\vec{\nabla} \times \overrightarrow{\mathbf{F}})
\end{aligned}
$$

then it is an easy matter to use (9) and (10) to show that

$$
\begin{aligned}
& \overrightarrow{\boldsymbol{\nabla}} \cdot\left(\frac{1}{r^{2}} \hat{\mathbf{r}}\right)=0, \\
& \overrightarrow{\boldsymbol{\nabla}} \times\left(\frac{1}{r \sin \theta} \hat{\boldsymbol{\phi}}\right)=\overrightarrow{\mathbf{0}}
\end{aligned}
$$

for instance by using $r^{2}=x^{2}+y^{2}+z^{2}$ and $r \sin \theta=\sqrt{x^{2}+y^{2}}$. But these are precisely (4) and (5); the hard work here is in deriving the initial formulas (9) and (10). This approach is closely related to the concept of covariant differentiation in differential geometry.

\section{Physics}

After defining the divergence and curl in terms of a Cartesian basis, an introductory mathematics course typically goes on to prove the divergence theorem and Stokes' theorem. If there is time-there often is not-a geometric interpretation is then provided through the formulas

$$
\begin{aligned}
& \vec{\nabla} \cdot \overrightarrow{\mathbf{F}}=\lim _{S \rightarrow 0} \frac{1}{\operatorname{Volume}(S)} \iint_{S} \overrightarrow{\mathbf{F}} \cdot d \overrightarrow{\mathbf{S}}, \\
& (\vec{\nabla} \times \overrightarrow{\mathbf{F}}) \cdot \hat{\mathbf{n}}=\lim _{C \rightarrow 0} \frac{1}{\operatorname{Area}(C)} \oint_{C} \overrightarrow{\mathbf{F}} \cdot d \overrightarrow{\mathbf{r}}
\end{aligned}
$$

which relate divergence and curl to flux and circulation, respectively.

Physicists often turn this around, and use these formulas to define the divergence and curl, thus turning the divergence theorem and Stokes' theorem into tautologies. These formulas are then used to compute the formulas for the divergence and curl in various coordinate systems. In spherical coordinates, for instance, this leads to formulas such as

$$
\vec{\nabla} \cdot\left(F^{r} \hat{\mathbf{r}}\right)=\frac{1}{r^{2}} \frac{\partial}{\partial r}\left(r^{2} F^{r}\right),
$$

$$
\overrightarrow{\boldsymbol{\nabla}} \times\left(F^{\phi} \hat{\boldsymbol{\phi}}\right)=\frac{1}{r \sin \theta} \frac{\partial}{\partial \theta}\left(\sin \theta F^{\phi}\right) \hat{\mathbf{r}}-\frac{1}{r} \frac{\partial}{\partial r}\left(r F^{\phi}\right) \hat{\boldsymbol{\theta}}
$$

from which (13) and (14) follow immediately. For a good, informal description of this approach, see Schey. ${ }^{5}$

\section{Orthogonal coordinates}

The preceding approach generalizes naturally to any orthogonal coordinate system, that is, one in which the three coordinate directions are everywhere orthogonal. Typical examples are rectangular, cylindrical, and spherical coordinates, but there are many more.

A general orthogonal coordinate system $(u, v, w)$ will have a line element of the form

$$
d s^{2}=f^{2} d u^{2}+g^{2} d v^{2}+h^{2} d w^{2} .
$$

If we denote the unit vector fields in the coordinate directions by $\{\hat{\mathbf{u}}, \hat{\mathbf{v}}, \hat{\mathbf{w}}\}$, then we can expand any vector field $\overrightarrow{\mathbf{F}}$ as

$$
\overrightarrow{\mathbf{F}}=F^{u} \hat{\mathbf{u}}+F^{v} \hat{\mathbf{v}}+F^{w} \hat{\mathbf{w}} .
$$

It is then a fairly simple computation ${ }^{6}$ to derive the general formulas

$$
\begin{aligned}
& \overrightarrow{\boldsymbol{\nabla}} \cdot \overrightarrow{\mathbf{F}}=\frac{1}{f g h} \frac{\partial}{\partial u}\left(g h F^{u}\right)+\cdots, \\
& \overrightarrow{\boldsymbol{\nabla}} \times \overrightarrow{\mathbf{F}}=\frac{1}{f g}\left[\frac{\partial}{\partial u}\left(g F^{v}\right)-\frac{\partial}{\partial v}\left(f F^{u}\right)\right] \hat{\mathbf{w}}+\cdots
\end{aligned}
$$

using the formulas (15) and (16).

These formulas can hardly be called obvious. The corresponding formula for the gradient is much more natural. Starting from the chain rule, in the form

$$
d k=\frac{\partial k}{\partial u} d u+\frac{\partial k}{\partial v} d v+\frac{\partial k}{\partial w} d w
$$

the all-important directional derivative, in the form

$$
d k=\vec{\nabla} k \cdot d \overrightarrow{\mathbf{r}}
$$

together with the "square root" of the line element (in the sense $d \overrightarrow{\mathbf{r}} \cdot d \overrightarrow{\mathbf{r}}=d s^{2}$ ), given by

$$
d \overrightarrow{\mathbf{r}}=f d u \hat{\mathbf{u}}+g d v \hat{\mathbf{v}}+h d w \hat{\mathbf{w}},
$$

we obtain

$$
\vec{\nabla} k=\frac{1}{f} \frac{\partial k}{\partial u} \hat{\mathbf{u}}+\frac{1}{g} \frac{\partial k}{\partial v} \hat{\mathbf{v}}+\frac{1}{h} \frac{\partial k}{\partial w} \hat{\mathbf{w}} .
$$

Examining (21) and (22), we see that there are special vector fields which are divergence or curl free, since

$$
\begin{aligned}
& \overrightarrow{\boldsymbol{\nabla}} \cdot \frac{\hat{\mathbf{u}}}{g h}=0, \\
& \overrightarrow{\boldsymbol{\nabla}} \times \frac{\hat{\mathbf{u}}}{f}=\overrightarrow{\mathbf{0}},
\end{aligned}
$$

and similarly for $\hat{\mathbf{v}}$ and $\hat{\mathbf{w}}$. These formulas can also be derived from the identities

$$
\begin{aligned}
& \vec{\nabla} \cdot(\vec{\nabla} \times \overrightarrow{\mathbf{F}})=0, \\
& \vec{\nabla} \times \vec{\nabla} f=\overrightarrow{\mathbf{0}},
\end{aligned}
$$

when one realizes that in orthogonal coordinates one has 


$$
\begin{aligned}
& \frac{\hat{\mathbf{u}}}{g h}=\vec{\nabla} v \times \vec{\nabla} w=\vec{\nabla} \times(v \vec{\nabla} w), \\
& \frac{\hat{\mathbf{u}}}{f}=\vec{\nabla} u .
\end{aligned}
$$

\section{ELECTROMAGNETIC CONIC SECTIONS}

\section{A. More product rules}

As discussed in Boas, ${ }^{7}$ the existence of a natural divergence-free basis along the lines of (27) can be used to reduce the computation of the divergence to the much simpler computation of the gradient. Similarly, the existence of a natural curl-free basis along the lines of (28) can be used to simplify the computation of the curl. In each case, this is accomplished using the appropriate product rule, (11) or (12), respectively. However, it is noteworthy that these two natural bases only agree in rectangular coordinates.

What if one could find a basis which was both divergenceand curl-free? In that case, one would never need to remember the formulas for the divergence and curl; all computations would reduce to the much simpler formula for the gradient.

Such a basis would also be of physical interest. A vector field which is both divergence- and curl-free solves Maxwell's vacuum equations, and can hence be interpreted as an electric or magnetic field. We are thus led to ask whether we can find a basis of electromagnetic fields.

We begin by considering several examples.

\section{B. Plane}

First of all, the rectangular basis $\{\hat{\mathbf{i}}, \hat{\mathbf{j}}, \hat{\mathbf{k}}\}$ is constant, and therefore, of course, both divergence- and curl-free. Each basis vector field must therefore describe an electromagnetic field. Which one? Consider an infinite parallel-plate capacitor, ${ }^{8}$ with infinite separation between the plates. If the plates have equal but opposite (uniform) charge densities, then there is a constant electric field orthogonal to the plates. If, instead, the plates have equal but opposite (uniform) current densities, then there is a constant magnetic field parallel to the plates (but orthogonal to the currents).

\section{Cylinder} by $^{9}$

Consider now the cylindrical coordinate system, defined

$$
\begin{gathered}
\sqrt{x^{2}+y^{2}}=s, \\
\tan ^{-1}\left(\frac{y}{x}\right)=\phi .
\end{gathered}
$$

Horizontal $(z=$ constant $)$ and vertical ( $\tan \phi=$ constant) slices through this coordinate system are shown in Fig. 1. Denoting the orthonormal basis for cylindrical coordinates as usual by $\{\hat{\mathbf{s}}, \hat{\boldsymbol{\phi}}, \hat{\mathbf{z}}\}$, we have $\hat{\mathbf{z}} \equiv \hat{\mathbf{k}}$, and thus this basis vector field is both divergence- and curl-free. But what about the other basis vectors?

The simplest cylindrical electromagnetic fields correspond to an infinite straight wire carrying either a uniform charge density or a uniform current density. It is straightforward to work out the corresponding fields: Up to scale factors, the electric field of the (positively) charged $z$ axis is
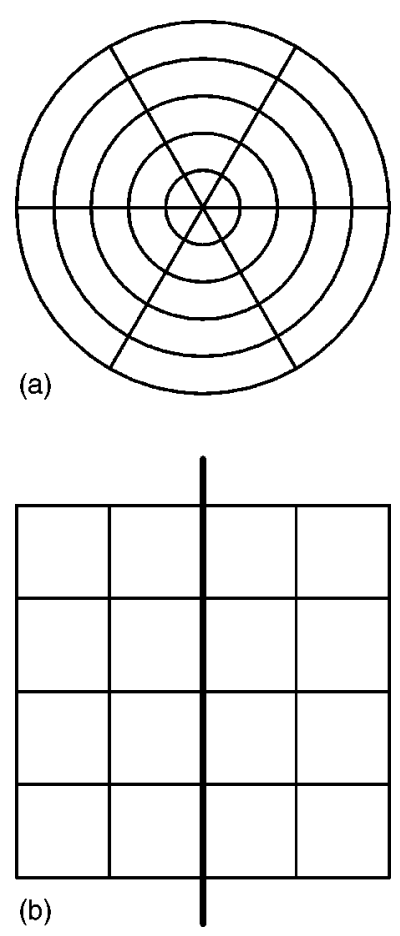

Fig. 1. (a) A horizontal slice of cylindrical coordinates, resulting in the usual polar coordinate grid. (b) A vertical slice of cylindrical coordinates, through the $z$ axis (shown as a heavy line).

$$
\overrightarrow{\mathbf{S}}=\frac{1}{s} \hat{\mathbf{S}}
$$

and the magnetic field of the (upward) current-carrying $z$ axis is

$$
\overrightarrow{\boldsymbol{\Phi}}=\frac{1}{s} \hat{\boldsymbol{\phi}} .
$$

Thus, an "electromagnetic" basis in this case is given by $\{\overrightarrow{\mathbf{S}}, \overrightarrow{\boldsymbol{\Phi}}, \hat{\mathbf{z}}\}$.

All of our remaining examples will be axially symmetric, and will thus have $\phi$ as a coordinate, $\hat{\boldsymbol{\phi}}$ as a basis vector field, and $\overrightarrow{\boldsymbol{\Phi}}$ as an electromagnetic basis vector field (although $s=\sqrt{x^{2}+y^{2}}$ will need to be expressed in terms of the given coordinates). We will omit further discussion of this case in (most of) the subsequent examples, and we will have no further use for horizontal slices analogous to (a) in Fig. 1.

\section{Sphere}

What about the other standard coordinate system, namely spherical coordinates, defined implicitly by

$$
\begin{aligned}
& \sqrt{x^{2}+y^{2}}=r \sin \theta, \\
& z=r \cos \theta
\end{aligned}
$$

(with $\phi$ as before), and shown in Fig. 2. The orthonormal basis for spherical coordinates is $\{\hat{\mathbf{r}}, \hat{\boldsymbol{\theta}}, \hat{\boldsymbol{\phi}}\}$, and we already know that

$$
\overrightarrow{\boldsymbol{\Phi}} \equiv \frac{1}{r \sin \theta} \hat{\boldsymbol{\phi}}
$$

is both divergence- and curl-free. 


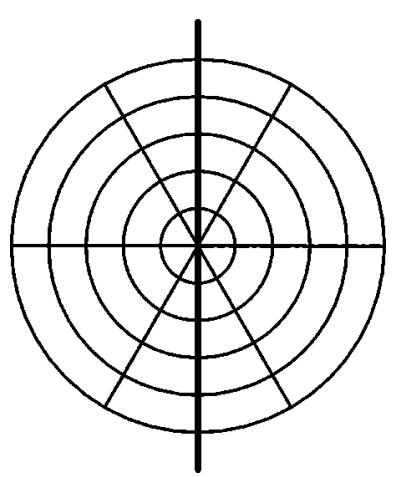

Fig. 2. A vertical slice of spherical coordinates, showing the $r \theta$ coordinate grid.

The only obvious spherical electromagnetic field is the electric field of a point charge, which is, up to a scale factor

$$
\overrightarrow{\mathbf{R}}=\frac{1}{r^{2}} \hat{\mathbf{r}}
$$

This solves part of the problem. But what electromagnetic field, if any, looks like $\hat{\boldsymbol{\theta}}$ ? Somewhat surprisingly, it turns out there is one, namely the electric field of two half-infinite uniform line charges, with equal but opposite charge densities, as shown in Fig. 3. Up to a scale factor, the resulting divergence-free and curl-free basis vector field is

$$
\overrightarrow{\boldsymbol{\Theta}}=\frac{1}{r \sin \theta} \hat{\boldsymbol{\theta}}
$$

and an electromagnetic basis is given by $\{\overrightarrow{\mathbf{R}}, \overrightarrow{\boldsymbol{\Theta}}, \overrightarrow{\boldsymbol{\Phi}}\}$.

\section{E. Spheroid and hyperboloid}

What about other, less common, orthogonal coordinate systems? Consider first prolate spheroidal ${ }^{10}$ coordinates, defined by

$$
\begin{aligned}
& \sqrt{x^{2}+y^{2}}=\sinh u \sin v, \\
& z=\cosh u \cos v
\end{aligned}
$$

as shown in Fig. 4. The relevant orthonormal basis vectors

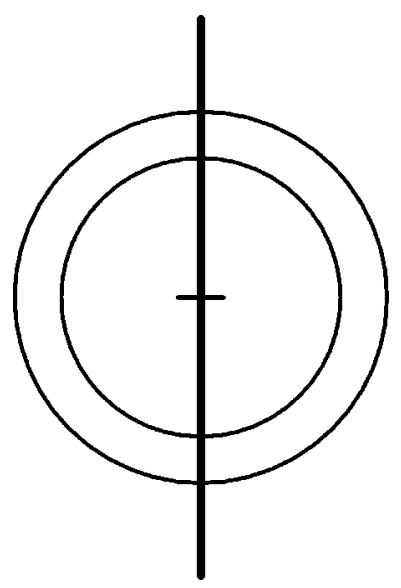

Fig. 3. A spherical electric field. If the positive $z$ axis is given a uniform positive charge density, and the negative $z$ axis is given an equal and opposite charge density, the resulting field lines are spherical, that is, in the $\hat{\boldsymbol{\theta}}$ direction.

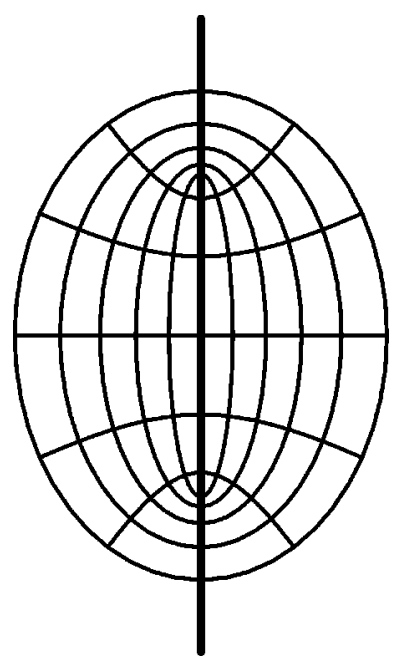

Fig. 4. A vertical slice of prolate spheroidal coordinates. The curves orthogonal to the ellipses are hyperbolas.

are $\hat{\mathbf{u}}$ and $\hat{\mathbf{v}}$; our goal is to find multiples of these which are both divergence- and curl-free, if possible.

With the wisdom of hindsight, that is, after having first computed the answer by brute force, it is clear that such vector fields do indeed exist. Consider the spherical model above, in which a multiple of $\hat{\boldsymbol{\theta}}$ was produced by two halfinfinite line charges which were joined at the origin. Separate the two instead by a finite distance, as shown in Fig. 5. The resulting electric field is just (proportional to)

$$
\overrightarrow{\mathbf{V}}=\frac{1}{\sin v \sqrt{\sinh ^{2} u+\sin ^{2} v}} \hat{\mathbf{v}}
$$

and is therefore spheroidal. Similarly, the electric field of the "missing" finite line segment is just (proportional to)

$$
\overrightarrow{\mathbf{U}}=\frac{1}{\sinh u \sqrt{\sinh ^{2} u+\sin ^{2} v}} \hat{\mathbf{u}}
$$

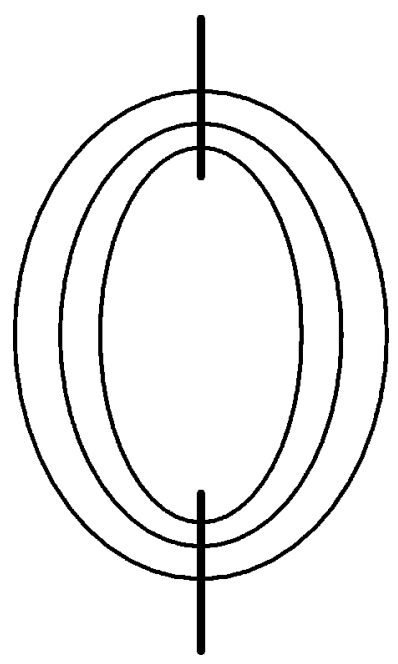

Fig. 5. A spheroidal electric field. If the oppositely charged half-lines in the spherical example are separated by a finite gap, the resulting field lines are spheroidal. 


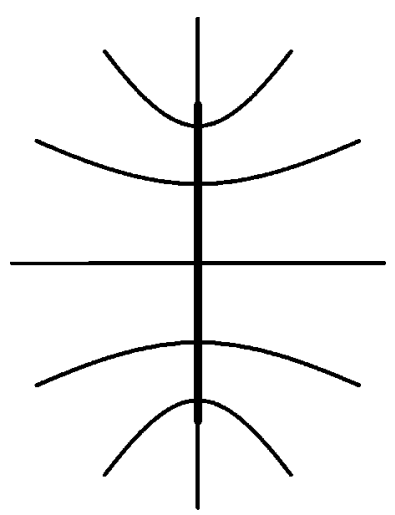

Fig. 6. A hyperboloidal electric field, the electric field of a uniformly charged line segment.

which is hyperboloidal, as shown in Fig. $6 .{ }^{11}$ An electromagnetic basis in this case is therefore given by $\{\overrightarrow{\mathbf{U}}, \overrightarrow{\mathbf{V}}, \overrightarrow{\boldsymbol{\Phi}}\}$.

\section{F. Paraboloid}

Moving right along, now consider parabolic coordinates, defined by

$$
\begin{aligned}
& \sqrt{x^{2}+y^{2}}=u v, \\
& z=\frac{1}{2}\left(u^{2}-v^{2}\right)
\end{aligned}
$$

and shown in Fig. 7. Do there exist multiples of $\hat{\mathbf{u}}$ and $\hat{\mathbf{v}}$ which are both divergence- and curl-free?

Again, with the wisdom of hindsight the answer is clearly yes. The electric field of a half-infinite, uniform line charge is shown in Fig. 8, corresponding to

$$
\begin{aligned}
& \overrightarrow{\mathbf{U}}=\frac{1}{u \sqrt{u^{2}+v^{2}}} \hat{\mathbf{u}}, \\
& \overrightarrow{\mathbf{V}}=\frac{1}{v \sqrt{u^{2}+v^{2}}} \hat{\mathbf{v}},
\end{aligned}
$$

respectively.

\section{G. Another hyperboloid}

Buoyed by our success, let us finally consider hyperboloidal (inverse paraboloidal) coordinates, defined by

$$
\begin{aligned}
& \sqrt{x^{2}+y^{2}}=\sqrt{\sqrt{u^{2}+v^{2}}-u}, \\
& z=\sqrt{\sqrt{u^{2}+v^{2}}+u},
\end{aligned}
$$

and shown in Fig. 9. We have

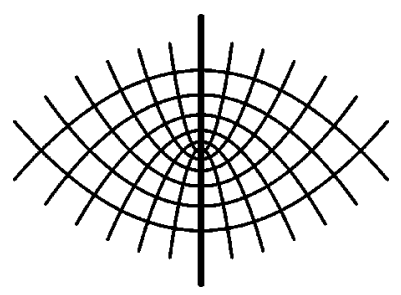

Fig. 7. A vertical slice of parabolic coordinates. Both families of orthogonal curves are parabolas.

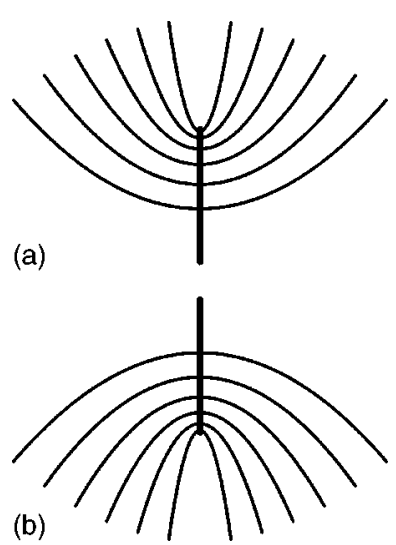

Fig. 8. Two paraboloidal electrical fields, namely the electric field of a half-infinite uniform line charge, along (a) the negative $z$ axis and (b) the positive $z$ axis.

$$
\begin{aligned}
\hat{\mathbf{u}} & =\frac{-x \hat{\mathbf{i}}-y \hat{\mathbf{j}}+z \hat{\mathbf{k}}}{\sqrt{x^{2}+y^{2}+z^{2}}}, \\
\hat{\mathbf{v}} & =\frac{z x \hat{\mathbf{i}}+z y \hat{\mathbf{j}}+\left(x^{2}+y^{2}\right) \hat{\mathbf{k}}}{\sqrt{x^{2}+y^{2}+z^{2}} \sqrt{x^{2}+y^{2}}}
\end{aligned}
$$

and we seek multiples of $\hat{\mathbf{u}}$ and $\hat{\mathbf{v}}$ which are both divergenceand curl-free.

There are none.

\section{H. General case}

So when does it work?

Given a vector field $\overrightarrow{\mathbf{G}}$, we ask whether there exists a function $\lambda$ such that $\lambda \overrightarrow{\mathbf{G}}$ is both divergence- and curl-free, that is, such that

$$
\begin{aligned}
& \vec{\nabla} \cdot \lambda \overrightarrow{\mathbf{G}}=0, \\
& \vec{\nabla} \times \lambda \overrightarrow{\mathbf{G}}=\overrightarrow{\mathbf{0}} .
\end{aligned}
$$

Using the product rules (11) and (12), we can rewrite these conditions as

$$
\begin{aligned}
& \vec{\nabla} \lambda \cdot \overrightarrow{\mathbf{G}}=-\lambda \vec{\nabla} \cdot \overrightarrow{\mathbf{G}}, \\
& \vec{\nabla} \lambda \times \overrightarrow{\mathbf{G}}=-\lambda \vec{\nabla} \times \overrightarrow{\mathbf{G}} .
\end{aligned}
$$

On the other hand, the identity

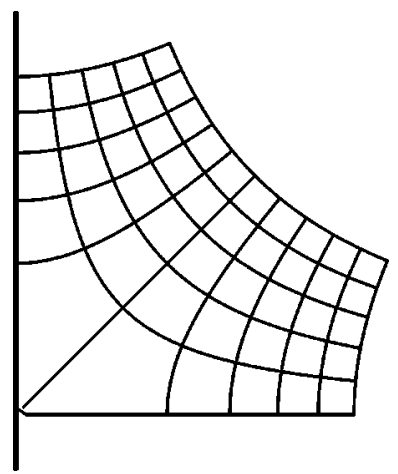

Fig. 9. A vertical slice of hyperboloidal coordinates. Both families of orthogonal curves are hyperbolas. 


$$
(\overrightarrow{\mathbf{u}} \times \overrightarrow{\mathbf{v}}) \times \overrightarrow{\mathbf{w}}=(\overrightarrow{\mathbf{u}} \cdot \overrightarrow{\mathbf{w}}) \overrightarrow{\mathbf{v}}-(\overrightarrow{\mathbf{v}} \cdot \overrightarrow{\mathbf{w}}) \overrightarrow{\mathbf{u}}
$$

leads to

$$
(\vec{\nabla} \lambda \times \overrightarrow{\mathbf{G}}) \times \overrightarrow{\mathbf{G}}=(\vec{\nabla} \lambda \cdot \overrightarrow{\mathbf{G}}) \overrightarrow{\mathbf{G}}-|\overrightarrow{\mathbf{G}}|^{2} \vec{\nabla} \lambda .
$$

Rearranging terms and using (56) and (57) then yields

$$
\begin{aligned}
\vec{\nabla} \lambda & =(\vec{\nabla} \lambda \cdot \overrightarrow{\mathbf{G}}) \frac{\overrightarrow{\mathbf{G}}}{|\overrightarrow{\mathbf{G}}|^{2}}-(\vec{\nabla} \lambda \times \overrightarrow{\mathbf{G}}) \times \frac{\overrightarrow{\mathbf{G}}}{|\overrightarrow{\mathbf{G}}|^{2}} \\
& =\lambda\left((\vec{\nabla} \times \overrightarrow{\mathbf{G}}) \times \frac{\overrightarrow{\mathbf{G}}}{|\overrightarrow{\mathbf{G}}|^{2}}-(\vec{\nabla} \cdot \overrightarrow{\mathbf{G}}) \frac{\overrightarrow{\mathbf{G}}}{|\overrightarrow{\mathbf{G}}|^{2}}\right) .
\end{aligned}
$$

Dividing (60) by $\lambda$ and taking the curl of both sides yields

$$
\overrightarrow{\mathbf{0}}=\vec{\nabla} \times\left((\vec{\nabla} \times \overrightarrow{\mathbf{G}}) \times \frac{\overrightarrow{\mathbf{G}}}{|\overrightarrow{\mathbf{G}}|^{2}}-(\vec{\nabla} \cdot \overrightarrow{\mathbf{G}}) \frac{\overrightarrow{\mathbf{G}}}{|\overrightarrow{\mathbf{G}}|^{2}}\right)
$$

since the left-hand side is the curl of $\vec{\nabla} \ln \lambda$. The necessary and sufficient condition that a suitable $\lambda$ exist is therefore (61); if $\lambda$ exists, then (61) is satisfied due to the identity (30), whereas if (61) is satisfied, then there exists a (local) potential function, which is $\ln \lambda$.

\section{DISCUSSION}

We have demonstrated a possible alternative way to compute the divergence and curl in certain standard cases. For instance, in spherical coordinates, one really need only remember that $\{\overrightarrow{\mathbf{R}}, \overrightarrow{\boldsymbol{\Theta}}, \overrightarrow{\boldsymbol{\Phi}}\}$ is an electromagnetic basis-ideally by recalling the corresponding electromagnetic fields. The divergence and curl are then easily computed from formulas like

$$
\begin{aligned}
& \vec{\nabla} \cdot(f \overrightarrow{\mathbf{R}})=\vec{\nabla} f \cdot \overrightarrow{\mathbf{R}}, \\
& \vec{\nabla} \times(f \overrightarrow{\mathbf{R}})=\vec{\nabla} f \times \overrightarrow{\mathbf{R}} .
\end{aligned}
$$

Yes, this requires knowing how to compute the gradient in spherical coordinates, but this can easily be rederived as needed from the geometrically obvious formula

$$
d \overrightarrow{\mathbf{r}}=d r \hat{\mathbf{r}}+r d \theta \hat{\boldsymbol{\theta}}+r \sin \theta d \boldsymbol{\phi} \hat{\boldsymbol{\phi}} .
$$

We have given several examples of orthogonal coordinates which admit an "electromagnetic basis." All of these examples are separable coordinates in the sense of Morse and Feschbach, ${ }^{12}$ that is, Laplace's equation is separable in these coordinates. It is straightforward to check that all 11 of the separable coordinate systems in Morse and Feschbach, ${ }^{12}$ all of which are special cases of ellipsoidal coordinates, admit an electromagnetic basis.

One might suspect that separable coordinates are the only ones which admit an electromagnetic basis. However, there are also nonseparable coordinates which admit an electromagnetic basis, an example being "logcoshcylindrical" coordinates, defined by

$$
\begin{aligned}
& x=\ln \left(\cosh ^{2} u-\sin ^{2} v\right), \\
& y=2 \tan ^{-1}(\tanh u \tan v), \\
& z=w .
\end{aligned}
$$

Turning to the general case, the condition (61) not only characterizes the vector fields $\overrightarrow{\mathbf{G}}$ which can be rescaled so as to be both divergence- and curl-free, it also provides an explicit algorithm for determining $\lambda$. There is another, simpler characterization, but without this property.

Requiring $\overrightarrow{\mathbf{F}}$ to be curl-free means that (locally)

$$
\overrightarrow{\mathbf{F}}=\vec{\nabla} f \text {. }
$$

In particular, since we are assuming $\overrightarrow{\mathbf{F}}=\lambda \overrightarrow{\mathbf{G}}$, this forces the original vector field $\overrightarrow{\mathbf{G}}$ to be orthogonal to the surfaces $\{f=$ constant $\}$. Thus, a necessary condition on $\overrightarrow{\mathbf{G}}$ is that it be hypersurface orthogonal. This condition is always satisfied for the examples considered here, constructed from a coordinate system.

The condition that $\overrightarrow{\mathbf{F}}$ be divergence-free imposes the further condition that

$$
\Delta f=0,
$$

so that $\overrightarrow{\mathbf{F}}$ must be the gradient of a harmonic function. Thus, the question of which coordinate systems admit basis vectors which can (all) be rescaled so as to be divergence- and curlfree is equivalent to the question of which coordinate systems can themselves be rescaled so as to be harmonic coordinates.

We conclude by noting that harmonic functions in two dimensions are closely related to analytic functions. A vector field $\overrightarrow{\mathbf{F}}=P \hat{\mathbf{i}}+Q \hat{\mathbf{j}}$ is divergence- and curl-free if, and only if, $P-i Q$ is analytic, since ${ }^{13}$

$$
\frac{\partial}{\partial \bar{z}}(P-i Q)=\frac{1}{2}(\vec{\nabla} \cdot \overrightarrow{\mathbf{F}}-i|\vec{\nabla} \times \overrightarrow{\mathbf{F}}|) .
$$

\section{ACKNOWLEDGMENTS}

It is a pleasure to thank Reed College for a colloquium invitation which got this project started. This material is based upon work supported by the National Science Foundation under Grants Nos. DUE-9653250 (Paradigms Project) and DUE-0088901 (Vector Calculus Bridging Project). This work has also been supported by the Oregon Collaborative for Excellence in the Preparation of Teachers (OCEPT) and by an L L Stewart Faculty Development Award from Oregon State University.

\footnotetext{
${ }^{a)}$ Electronic mail: tevian@math.orst.edu

${ }^{b)}$ Electronic mail: corinne@ physics.orst.edu

${ }^{1}$ Jason W. Dunn and Julius Barbane, "One model for an integrated math/ physics course focusing on electricity and magnetism and related calculus topics," Am. J. Phys. 68, 749-757 (2000).

${ }^{2}$ Tevian Dray and Corinne A. Manogue, "The vector calculus gap: mathematics $\neq$ physics," PRIMUS 9, 21-28 (1999).

${ }^{3}$ For the record, while a traditional course in multivariable or vector calculus will certainly discuss polar, cylindrical, and spherical coordinates, vectors will most likely be expressed exclusively in terms of their rectangular components.

${ }^{4}$ Tevian Dray and Corinne A. Manogue, "Using differentials to bridge the vector calculus gap," College Math. J. (to appear).

${ }^{5}$ H. M. Schey, div, grad, curl, and all that, 3rd ed. (Norton, New York, 1997).

${ }^{6}$ David J. Griffiths, Introduction to Electrodynamics, 3rd ed. (Prentice-Hall, New York, 1999).

${ }^{7}$ Mary L. Boas, Mathematical Methods in the Physical Sciences, 2nd ed. (Wiley, New York, 1983).

${ }^{8}$ One plate is in fact sufficient. The advantage of two plates is that the field vanishes outside the capacitor.

${ }^{9}$ We use $\phi$ rather than $\theta$ for compatibility with our later examples, and $s$
} 
rather than $r$ to avoid confusion with spherical coordinates.

${ }^{10} \mathrm{~A}$ spheroid is an ellipsoid with two axes of the same length.

${ }^{11}$ It is instructive to consider this latter example as a "stretched out" point charge.
${ }^{12}$ Philip M. Morse and Herman Feshbach, Methods of Theoretical Physics (McGraw-Hill, New York, 1953), Chap. 5.

${ }^{13} \mathrm{~A}$ similar statement can be made in three dimensions, using quaternions in place of the complex numbers. 